\title{
Comparison of the ease of laryngeal mask airway ProSeal insertion and the fiberoptic scoring according to the head position and the presence of a difficult airway
}

\author{
Joo Hyun Jun, Hee Jung Baik, Jong Hak Kim, Youn Jin Kim, and Ri-Na Chang \\ Department of Anesthesiology and Pain Medicine, School of Medicine, Ewha Womans University, Seoul, Korea
}

Background: The sniffing position is recommended for conventional laryngeal mask airway (LMA) insertion. However, there has been a high success rate of LMA insertion with the head in the neutral position. The effect of a difficult airway on the ease of LMA insertion is not clear. In this study, we compared the ease of LMA ProSeal ${ }^{\mathrm{TM}}$ (PLMA) insertion and the fiberoptic scoring according to the head position and the presence of a difficult airway.

Methods: After obtaining informed consent from the subjects, we enrolled 144 adult patients (age range: 18-65) with an ASA physical status 1 or 2 . After evaluation of the airway, all the patients were grouped into the EA (easy airway) group $(n=68)$ and the DA (difficult airway) group $(n=76)$. According to the head position, each group was divided into the EA-SE (extension) group ( $\mathrm{n}=35)$, the EA-SN (sniffing) group $(\mathrm{n}=33)$, the DA-SE group $(\mathrm{n}=39)$ and the DASN group $(n=37)$. The success rate and insertion time at the first attempt were evaluated. The position of the PLMA was fiberoptically scored from the mask aperture of the airway tube in the original head position. After the head position was changed to the sniffing and neutral positions in the SE and SN group, respectively, the position of PLMA was re-evaluated fiberoptically.

Results: The success rate and insertion time at the first attempt and the fiberoptic score showed no significant difference among the groups. After head position was changed, there were no significant changes in the fiberopitc scores.

Conclusions: A difficult airway and the head position had no influence on the ease of PLMA insertion and the fiberopic score. Therefore, the head position can be selected according to the individual patient's situation. (Korean J Anesthesiol 2011; 60: 244-249)

Key Words: Diffcult airway, Laryngeal mask airway, Neutral position, Sniffing position.

Received: September 30, 2010. Revised: October 13, 2010. Accepted: October 13, 2010.

Corresponding author: Hee Jung Baik, M.D., Department of Anesthesiology and Pain Medicine, School of Medicine, Ewha Womans University, 911-1, Mok 5-dong, Yangcheon-gu, Seoul 158-710, Korea. Tel: 82-2-2650-2868, Fax: 82-2-2655-2924, E-mail: baikhj@ewha.ac.kr (C) This is an open-access article distributed under the terms of the Creative Commons Attribution Non-Commercial License (http:// creativecommons.org/licenses/by-nc/3.0/), which permits unrestricted non-commercial use, distribution, and reproduction in any medium, provided the original work is properly cited. 


\section{Introduction}

A laryngeal mask airway (LMA) is frequently used not only as the airway device for anesthesia in elective surgery, but also for a difficult airway and as an essential airway device for cardiopulmonary resuscitation (CPR). Brain was the designer of the LMA, and he claimed that it could easily be inserted in cases with a difficult airway [1].

The "sniffing position" (i.e., the neck flexed and head extended by means of a pillow) is used during conventional tracheal intubation using direct laryngoscopy. This position eases tracheal intubation because the three axes, namely, the axis of the mouth, the pharyngeal axis and the laryngeal axis, are put in almost a straight line. Boidin stated that the main cause of upper airway obstruction was not the tongue but the epiglottis, and that head elevation with an $8 \mathrm{~cm}$-high pillow raised the epiglottis from the posterior pharyngeal wall and opened the upper airway. In this case, simple head extension could thoroughly open the airway [2]. However, Adnet et al. objected to the "three-axis alignment theory" of the airway structure in their study, in which they observed the magnetic resonance imaging of patients with normal airway anatomy [3]. They reported that the sniffing position had an advantage to simple head extension only for the cases of obesity and those cases with limited head extension [4]. The standard LMA insertion technique involves the sniffing position [5], but Brimacombe and Berry reported there was no significant difference in the success rate of insertion when they compared the sniffing position with the neutral position [6]. They also stated that further studies were needed since the modified Mallampati classification dose not seem to help predict the ease of LMA insertion or the fiberoptic positioning of the LMA [7].

However, those previous studies considered only one factor between head position and the presence of a difficult airway during LMA insertion, and the anatomic structure that caused difficult airway could not be predicted in a comprehensive manner since the studies did not consider predictors other than the modified Mallampati classification [6,7]. In this study we sought to compare the ease of LMA ProSeal ${ }^{\mathrm{TM}}$ (PLMA) insertion and the fiberopitc score according to the head position and the presence of difficult airway based on the airway score for predicting difficult intubation.

\section{Materials and Methods}

One hundred forty four adult patients aged between 18-65 with an ASA physical status of 1 or 2 and who were undergoing elective surgery under general anesthesia were the subject of our study. Our ethics committee approved this study and we obtained written, informed consent from all the patients. Patients were excluded if they had a history of cervical spine injury or they needed awake fiberopic intubation due to suspected anatomic abnormalities in the airway, or if they had severe cardiovascular diseases, the possibility of gastroesophageal reflux or poor dentition that might be damaged during the study.

None of the patients were premedicated, and they all underwent airway assessment for seven items such as the interincisor gap, the modified Mallampati classification, the thyromental distance, head neck movement [8], a history of difficult tracheal intubation, buck teeth and an upper lip bite test (ULBT) [9] before the induction of anesthesia on arrival to the operating room. The seven items were each assessed by a score of 0,1 or 2 with their sum being the total airway score. Patients with a score $\leq 2$ were classified into the EA (easy airway) group ( $\mathrm{n}=68)$, and those with a score $\geq 3$ were classified as the DA (difficult airway) group ( $\mathrm{n}=76$ ) (Table 1). According to the head position on insertion, the simple head extension was classified into the SE (simple extension) group, and the sniffing position where the neck was flexed and the head was extended by means of a $8 \mathrm{~cm}$-high pillow, was classified as the SN (sniffing) group. Therefore, the patients were classified into four groups: the EA-SE group $(\mathrm{n}=35)$, the EA-SN group $(\mathrm{n}=33)$, the DA-SE group $(n=39)$ and the DA-SN group $(n=37)$. The demographic data is shown in Table 2.

Preoxygenation with $100 \%$ oxygen was performed in all

Table 1. Preoperative Assessment of the Airway Score for Predicting Difficult Intubation

\begin{tabular}{|c|c|c|c|}
\hline & 0 & 1 & 2 \\
\hline 1. Interincisor gap & $>5 \mathrm{~cm}$ & $5-4 \mathrm{~cm}$ & $<4 \mathrm{~cm}$ \\
\hline 2. Modified Mallampati & Class I, II & Class III & Class IV \\
\hline 3. Thyromental distance & $>6.5 \mathrm{~cm}$ & $6.5-6.0 \mathrm{~cm}$ & $<6 \mathrm{~cm}$ \\
\hline 4. Head/Neck movement & $>90^{\circ}$ & $=90^{\circ}$ & $<90^{\circ}$ \\
\hline 5. History of D/I & No & Questionable & Definite \\
\hline 6. Buck teeth & No & Mild & Moderate \\
\hline 7. ULBT & $\begin{array}{l}\text { Lower incisors can hide the } \\
\text { mucosa of the upper lip }\end{array}$ & $\begin{array}{l}\text { Lower incisors partially hide } \\
\text { the mucosa of the upper lip }\end{array}$ & $\begin{array}{l}\text { Lower incisors unable to touch } \\
\text { the mucosa of the upper lip }\end{array}$ \\
\hline
\end{tabular}

$\mathrm{D} / \mathrm{I}$ : difficult intubation, ULBT: upper lip bite test, total airway score $\geq 3$ : difficult intubation predicted. 
Table 2. Demographic Data

\begin{tabular}{lcccc}
\hline & \multicolumn{3}{c}{ EA group } & \multicolumn{2}{c}{ DA group } \\
\cline { 2 - 5 } & EA-SE & EA-SN & DA-SE & DA-SN \\
$(\mathrm{n}=35)$ & $(\mathrm{n}=33)$ & $46.7 \pm 12.4$ & $41.9 \pm 9.3$ \\
Age $(\mathrm{yr})$ & $38.0 \pm 13.3$ & $38.1 \pm 15.4$ & $23 / 16$ & $17 / 20$ \\
Gender $(\mathrm{M} / \mathrm{F})$ & $21 / 14$ & $17 / 16$ & $163.7 \pm 10.5$ & $161.0 \pm 8.4$ \\
Height $(\mathrm{cm})$ & $168.1 \pm 9.3$ & $166.1 \pm 8.3$ & $63.6 \pm 8.7$ & $63.2 \pm 12.3$ \\
Weight $(\mathrm{kg})$ & $65.5 \pm 11.5$ & $66.0 \pm 12.2$ & $23.8 \pm 3.2$ & $24.1 \pm 3.3$ \\
BMI $\left(\mathrm{kg} / \mathrm{m}^{2}\right)$ & $23.1 \pm 3.1$ & $24.0 \pm 4.3$ & \\
\hline
\end{tabular}

Values are mean \pm SD or the number of patients. EA: easy airway, DA: difficult airway, SE: simple head extension, SN: sniffing position, BMI: body mass index.

Table 3. Proposed Fiberoptic Scoring System

\begin{tabular}{cl}
\hline Score & \multicolumn{1}{c}{ View } \\
\hline 4 & Only cords seen \\
3 & Cords plus posterior epiglottis seen \\
2 & Cords plus anterior epiglottis seen \\
1 & Cords not seen, but function adequate \\
0 & Cords not seen, failure to function \\
\hline
\end{tabular}

Fiberoptic scope position: just proximal to the mask aperture bars.

patients for two minutes. Anesthesia was induced with fentanyl $1 \mu \mathrm{g} / \mathrm{kg}$ and midazolam $0.04 \mathrm{mg} / \mathrm{kg}$ followed by propofol $2 \mathrm{mg} /$ $\mathrm{kg}$. Maintenance of anesthesia was achieved with oxygen and a $1 \%$ propofol continuous infusion $(6 \mathrm{mg} / \mathrm{kg} / \mathrm{hr})$. After loss of consciousness, neuromuscular relaxation was achieved with rocuronium $0.3 \mathrm{mg} / \mathrm{kg}$. After 3-minutes of controlled ventilation with $100 \%$ oxygen $6 \mathrm{~L} / \mathrm{min}$ via a facemask, an experienced researcher for PLMA (LMA ProSeal ${ }^{\mathrm{TM}}$, LMA North America Inc., San Diego, CA, USA) and who had not participated in the airway assessment performed the PLMA insertion. The PLMA size was chosen according to the patient's weight: $<50$ kg: size 3, 50-70 kg: size 4 and $>70 \mathrm{~kg}$ : size 5 . After inserting the PLMA, the cuff was inflated with a minimum volume that had no gas leak by the 'just seal' method. The success of PLMA insertion was judged as no gas leak in the mouth under an airway pressure of $20 \mathrm{cmH}_{2} \mathrm{O}$, breathing sounds were uniformly auscultated in both lungs, there was no sound of air influx to the gastrointestinal tract and the capnogram showed a normal square wave. Insertion was considered a failure when the PLMA was not advanced to the pharynx, or if there was a severe gas leak through the mouth or the drain tube, normal lung ventilation was not observed, or the end tidal carbon dioxide pressure $\left(\mathrm{P}_{\mathrm{ET}} \mathrm{CO}_{2}\right)$ was not normal on ventilation after completing the insertion. If insertion was failed, insertion was re-tried after 1-minute of positive pressure-controlled ventilation via the face mask. Unsuccessful insertion after three attempts was regarded as a failure.

In all patients, the number of insertion attempts, the success/ failure of the three attempts and the insertion time of the first attempt were recorded. The insertion time of the first attempt was estimated as the time from the operator opening the patient's mouth to removing his hand after PLMA insertion into the pharynx. The ease of insertion was classified as "easy" if the first attempt was successful, "difficult" in cases with success on the second or the third attempt and "failure" in cases where all three attempts had failed. If ventilation was appropriate or possible, then the PLMA was fixed at that position, auscultation was done at the existing head position to determine whether both lungs were well ventilated and whether a grunting sound was heard from the neck, and thereafter the fiberoptic position was observed (LF-GP, Olympus, Japan) from the mask aperture bar. After the head position was changed into the sniffing and neutral positions in the SE and SN groups, respectively, the position was re-evaluated for breathing sounds in both lungs, a grunting sound from the neck and changes in the fiberoptic position. The fiberoptic position was scored from the mask aperture bar as 4 : only the vocal cords were seen, 3: the vocal cords plus the posterior epiglottis were seen, 2: the vocal cords plus the anterior epiglottis were seen, 1 : the vocal cords were not seen, but adequate function and 0 : the vocal cords were not seen, and there was failure to function (Table 3) [10].

Sample size calculation was done by G* Power (Ver 3.2.1, Germany). Through the preliminary tests of 5 patients per each group, the median insertion time of the first attempt was calculated as 11.7 seconds in the EA-SE group, 10.3 seconds in the EA-SN group, 10.0 seconds in the DA-SE group and 7.0 seconds in the DA-SN group. The total sample size was set as 100 calculated from an effect size of 0.34 , a power of $80 \%$, an $\alpha$ of 0.05 where the standard deviation of four groups was five seconds. Assuming a $20 \%$ dropout rate, the total sample size was set at 120 (a minimum of over 30 per each group). The age, height, weight, the BMI, and the insertion time of the first attempt of the patients were expressed as mean \pm standard deviation. The success of PLMA insertion after the first attempt, the success/failure of the three attempts and the fiberoptic scoring were expressed as frequencies. SPSS (Ver 17.0) was used for all the statistical analyses: chi-square tests 
were used for comparisons of the four groups for the success of the first attempt, the success/failure of the three attempts and the fiberoptic scoring. One-way analysis of variance (ANOVA) was used for comparative analysis among the groups for the insertion time of the first attempt for the 122 patients with a successful first insertion, and two-way ANOVA was performed to analyze the factors, the head position and a difficult airway with regard to their influence on the insertion time of the first attempt and whether they had interaction. P values $<0.05$ were considered statistically significant.

\section{Results}

The ease of insertion was not statistically different among the four groups. The success rate of the first attempt was $85.0 \%$ in the EA-SE group, $87.9 \%$ in the EA-SN group, $82.0 \%$ in the DASE group and $89.2 \%$ in the DA-SN group. The total success rate after three attempts was $94-100 \%$ in the four groups, with no significant difference $(\mathrm{P}>0.05)$ (Table 4$)$. The insertion time of the first attempt was $9.2 \pm 5.1 \mathrm{~s}$ in the EA-SE group, $7.8 \pm 4.9$ $\mathrm{s}$ in the EA-SN group, $9.2 \pm 5.4 \mathrm{~s}$ in the DA-SE group and 8.6 $\pm 4.9 \mathrm{~s}$ in the DA-SN group, with no statistical difference ( $\mathrm{P}$ $>0.05$ ) among the four groups. On the two-way ANOVA for factor analysis, the head position and a difficult airway did not influence the insertion time of the first attempt $(\mathrm{P}>0.05)$, and there was no interaction between the factors $(\mathrm{P}>0.05)$ (Table 5). In the ideal cases with a score $\geq 2$ where the vocal cords were seen, the fiberoptic position according to the head position and the presence of a difficult airway was not statistically different (P > 0.05) (Table 4). After changing the head position, 3 EASE patients, 5 EA-SN patients, 4 DA-SE patients and 2 DA-SN patients showed an aggravated fiberoptic score, while one patient in the DA-SE and DA-SN groups, respectively, had an improved fiberoptic score. Yet, the changes in the score were not statistically significant $(\mathrm{P}>0.05$ ) (Table 6). Moreover, 2 patients in the EA-SE group, 2 in the EA-SN group, 5 in the DA-

Table 4. Ease of Insertion and the Fiberoptic Score

\begin{tabular}{lccccc}
\hline & \multicolumn{3}{c}{ EA } & \multicolumn{2}{c}{ DA } \\
\cline { 2 - 3 } \cline { 5 - 6 } & $\begin{array}{c}\text { EA-SE } \\
(\mathrm{n}=35)\end{array}$ & $\begin{array}{c}\text { EA-SN } \\
(\mathrm{n}=33)\end{array}$ & & $\begin{array}{c}\text { DA-SE } \\
(\mathrm{n}=39)\end{array}$ & $\begin{array}{c}\text { DA-SN } \\
(\mathrm{n}=37)\end{array}$ \\
\hline Easy & $28(85.0 \%)$ & $29(87.9 \%)$ & $32(82.0 \%)$ & $33(89.2 \%)$ \\
Difficult & $5(14.3 \%)$ & $4(12.1 \%)$ & $6(15.4 \%)$ & $2(5.4 \%)$ \\
Failure & $2(5.7 \%)$ & 0 & & $1(2.6 \%)$ & $2(5.4 \%)$ \\
Success & $33(94.3 \%)$ & $33(100 \%)$ & $38(97.4 \%)$ & $35(94.6 \%)$ \\
FS $\geq 2$ & $32(91.4 \%)$ & $31(93.9 \%)$ & $34(87.2 \%)$ & $33(89.2 \%)$ \\
FS $\leq 1$ & $3(8.6 \%)$ & $2(6.1 \%)$ & $5(12.8 \%)$ & $4(10.8 \%)$ \\
\hline
\end{tabular}

Values are the number of patients. Easy: success at the first insertion attempt, Difficult: success at the 2nd or 3rd attempt, Failure: fail of insertion at more than the 3rd attempt, Success: total success of insertion, FS: Fiberoptic score.
SE group and 4 in the DA-SN group had grunting sounds and this did not disappear after changing the head position.

\section{Discussion}

The success rate at the first attempt, the insertion time of the first attempt and the fiberoptic score after insertion were not statistically different among the four groups. Changing the head position after insertion did not significantly change the fiberoptic score.

It is known that the sniffing position is ideal for conventional tracheal intubation with direct laryngoscopy [11,12]. We expected that this position made it easier to advance the PLMA into the pharynx than simple head extension during insertion. However, in this study, there was no significant difference in the ease of insertion and the success rate between the sniffing and simple extension positions for the EA group. This result coincides with the result of Brimacombe and Berry who reported that there was no difference in the rate of successful insertion between the sniffing and neutral positions [6]. However, since there was a report that the sniffing position was beneficial only for the obese and those with restricted head extension [4], we expected that unlike for the EA group, sniffing position for the DA group would be easier for PLMA insertion than simple head extension. Yet, the DA group

Table 5. Insertion Time at the First Attempt According to the Airway and the Head Position and their Interaction Effect $(n=122)$

\begin{tabular}{llc}
\hline \multicolumn{1}{c}{ Group } & $\begin{array}{c}\text { Insertion time at the } \\
\text { first attempt }(\mathrm{sec})\end{array}$ \\
\hline Airway & EA $(\mathrm{n}=57)$ & $8.5 \pm 5.0$ \\
Position & DA $(\mathrm{n}=65)$ & $8.9 \pm 5.1$ \\
Airway*Position & SE $(\mathrm{n}=60)$ & $9.2 \pm 5.2$ \\
& SN $(\mathrm{n}=62)$ & $8.2 \pm 4.9$ \\
& EA-SE $(\mathrm{n}=28)$ & $9.2 \pm 5.1$ \\
& EA-SN $(\mathrm{n}=29)$ & $7.8 \pm 4.9$ \\
& DA-SE $(\mathrm{n}=32)$ & $9.2 \pm 5.4$ \\
& DA-SN $(\mathrm{n}=33)$ & $8.6 \pm 4.9$ \\
\hline
\end{tabular}

Values are mean \pm SD or the number of patients. EA: easy airway, DA: difficult airway, SE: simple head extension, SN: sniffing position.

Table 6. Fiberoptic Score after Changing the Head Position

\begin{tabular}{lcccc}
\hline \multirow{2}{*}{ EA group } & & Better & Nc & Worse \\
& EA-SE & 0 & 32 & 3 \\
\multirow{5}{*}{ DA group } & EA-SN & 0 & 28 & 5 \\
& DA-SE & 1 & 34 & 4 \\
& DA-SN & 1 & 34 & 2 \\
& Total & 2 & 128 & 14 \\
\hline
\end{tabular}

Values are the number of patients. Better: improvemet after changing the head position, Nc: no change after changing the head position, Worse: aggravation after changing the head position. 
showed no difference in the ease of insertion or the success rate according to the head position. It can be presumed that our different results may have been due to the difference in tracheal intubation with direct laryngoscopy and PLMA insertion, and to the fact that none of the subjects in this study had a BMI over 30 $\mathrm{kg} / \mathrm{m}^{2}$.

According to Brain, intubation difficulties were associated with an apparently anteriorly placed larynx. In contrast, the patients who have difficulty with insertion of the LMA have an apparently posteriorly placed larynx, which tends to block the downward progress of the tip of the mask. The more anterior the larynx, the easier it is to insert the LMA behind it. Thus, when intubation is unusually difficult, use of the LMA may be easy [1]. However, Brimacombe and Berry reported that the modified Mallampati classification, which was used for predicting a difficult airway, did not help to predict the ease of LMA insertion and the fiberoptic scoring [7]. In contrast, another study reported that as the modified Mallampati classification increased, tracheal intubation became more difficult and securing the airway by the LMA also became difficult [13]. Moreover, the modified Mallampati classification can predict a difficult airway according to the tongue and pharynx structure, but it cannot predict a difficult airway according to extension of the atlas occipital joint and the mandibular space. Yet, the airway score, which was used in the study of Lee et al., is a test method to predict difficult tracheal intubation with more accuracy, sensitivity and specificity than the modified Mallampati classification [14]. Therefore, in this study, we performed airway assessment for seven items based on the airway score of Lee et al., and we added ULBT to comprehensively predict the anatomic structure that causes a difficult airway, and we estimated the seven items as a score of 0,1 or 2 , respectively, with their sum being the total airway score. We then classified the patients with a score $\leq 2$ into the EA group and the patients with a score $\geq 3$ into the DA group for our study (Table 2).

Brimacombe and Berry did not consider the predictors of a difficult airway in their study of the ease of LMA insertion according to the head position [6], and the head position on insertion and many airway predictors other than the modified Mallampati classification were not considered in another study on the ease of LMA insertion according to the presence of a difficult airway [7]. The results of our current study are thought to be very meaningful: because the insertion time of the first attempt, the rate of successful insertion, the changes in the fiberoptic score according to the head position and the presence of a difficult airway based on the airway score predicting difficult intubation were observed.

In this study, the success rate of PLMA insertion at the first attempt was $85.0 \%$ in the EA-SE group, $87.9 \%$ in the EA-SN group, $82.0 \%$ in the DA-SE group and $89.2 \%$ in the DA-SN group, which are similar to the $86.7 \%$ success rate of PLMA insertion at the first attempt in the study by Brimacombe and Keller [15]. The success rate over three attempts was $94-100 \%$ in the four groups, which was also similar to the result of Brimacombe and Keller and that of Figueredo et al. $[15,16]$. The insertion time of the first attempt was $9.7 \mathrm{~s}$ in the EA-SE group, $7.8 \mathrm{~s}$ in the EA-SN group, $9.3 \mathrm{~s}$ in the DA-SE group and $8.5 \mathrm{~s}$ in the DA-SN group, which were shorter than the $21.6 \mathrm{~s}$ in the results of Kim et al.'s study [17]. We can speculate this difference may have been caused by the method of insertion, the method of estimating the time and the operator's skill. In this study, the frequency of vocal cord visibility (a fiberoptic score $\geq 2$ ) was $87.2-93.9 \%$ in all four groups, which was similar to the results of Brimacombe and Keller (93.3\%) [15]. However, when they compared the ease of LMA insertion and PLMA insertion, the LMA had a better success rate of insertion at the first attempt and a better fiberoptic score than that of the PLMA [15]. Therefore, another study should be carried out that will focus on how head position and the presence of a difficult airway may influence the ease and fiberoptic scoring of conventional LMA insertion, other than just PLMA insertion.

In conclusion, this study revealed the head position on insertion and the presence of a difficult airway did not influence the ease of PLMA insertion or the fiberoptic scoring. Further, changes in the head position after PLMA insertion did not influence the fiberoptic scoring. Therefore, it is thought that the PLMA is an alternative airway device that noninvasively secures the airway when intubation is difficult, that the head position should be chosen with considering the presence of cervical damage and the dental status of the patients on PLMA insertion, and that the PLMA can be properly inserted in both the sniffing position and with simple head extension.

\section{References}

1. Brain AI. Three cases of difficult intubation overcome by the laryngeal mask airway. Anaesthesia 1985; 40: 353-5.

2. Boidin MP. Airway patency in the unconscious patient. Br J Anaesth 1985; 57: 306-10.

3. Adnet F, Borron SW, Dumas JL, Lapostolle F, Cupa M, Lapandry C. Study of the "sniffing position" by magnetic resonance imaging. Anesthesiology 2001; 94: 83-6.

4. Adnet F, Baillard C, Borron SW, Denantes C, Lefebvre L, Galinski $\mathrm{M}$, et al. Randomized study comparing the "sniffing position" with simple head extension for laryngoscopic view in elective surgery patients. Anesthesiology 2001; 95: 836-41.

5. Brain AI. The Laryngeal mask-a new concept in airway management. Br J Anaesth 1983; 55: 801-5.

6. Brimacombe J, Berry A. Laryngeal mask airway insertion. A comparison of the standard versus neutral position in normal patients with a view to its use in cervical spine instability. Anaesthesia 1993; 
48: $670-1$.

7. Brimacombe J, Berry A. Mallampati classification and laryngeal mask airway insertion. Anaesthesia 1993; 48: 347.

8. Wilson ME, Spiegelhalter D, Robertson JA, Lesser P. Predicting difficult intubation. Br J Anaesth 1988; 61: 211-6.

9. Khan ZH, Kashfi A, Ebrahimkhani E. A comparison of the upper lip bite test (a simple new technique) with modified Mallampati classification in predicting difficulty in endotracheal intubation: a prospective blinded study. Anesth Analg 2003; 96: 595-9.

10. Brimacombe J, Berry A. A proposed fiber-optic scoring system to standardize the assessment of laryngeal mask airway position. Anesth Analg 1993; 76: 457.

11. Horton WA, Fahy L, Charters P. Defining a standard intubating position using "angle finder". Br J Anaesth 1989; 62: 6-12.

12. Takenaka I, Aoyama K, Iwagaki T, Ishimura H, Kadoya T. The sniffing position provides greater occipito-atlanto-axial angulation than simple head extension: a radiological study. Can J Anaesth 2007; 54: 129-33.
13. McCrory CR, Moriarty DC. Laryngeal mask airway positioning is related to Mallampati grading in adults. Anesth Analg 1995; 81: 1001-4.

14. Lee SM, Kim DS, Ryu SJ, Chang TH, Kim SH, Kim KH. The evaluation of predictability of difficult intubation using upper lip bite test. Korean J Anesthesiol 2005; 49: 287-92.

15. Brimacombe J, Keller C. The ProSeal laryngeal mask airway: a randomized, crossover study with the standard laryngeal mask airway in paralyzed, anesthetized patients. Anesthesiology 2000; 93: 104-9.

16. Figueredo E, Martínez M, Pintanel T. A comparison of the ProSeal laryngeal mask and the laryngeal tube in spontaneously breathing anesthetized patients. Anesth Analg 2003; 96: 600-5.

17. Kim YY, Lee SK, Yi JS. Comparison between the index finger insertion and catheter-guided insertion techniques for airway placement of a ProSeal laryngeal mask by inexperienced personnel. Korean J Anesthesiol 2009; 56: 634-8. 